\title{
An Innovative Miniscrew-Based Jig for Unilateral Total Arch Mesialization
}

\author{
Siamak Hemmatpour ${ }^{1}$ (D), Golnaz Nahvi ${ }^{2, *(D)}$, Mehdi Oonchi ${ }^{3}$ (D) ${ }^{\text {Shahryar Karami }}{ }^{4}$ (D) \\ Department of Orthodontics, School of Dentistry, Tehran medical sciences, Islamic Azad University, Tehran, Iran \\ Department of Orthodontics, School of Dentistry, Shahid Beheshti University of Medical Sciences, Tehran, Iran \\ Faculty of Dentistry, University of British Columbia, Canada \\ 4 Department of Orthodontics, School of dentistry, Tehran medical sciences, Islamic Azad University, Tehran, Iran \\ * Correspondence: golnaznahvi@gmail.com;
}

Scopus Author ID 56082419000

Received: 20.08.2020; Revised: 12.09.2020; Accepted: 14.09.2020; Published: 15.09.2020

\begin{abstract}
To describe the management of a case with skewed dental arches and midlines deviation using a novel approach based on temporary anchorage devices (TADs) to derogate the deviated arches by unilateral total arch mesialization. This study presents the treatment course of a 25 -year-old man undergone a previous improper orthodontic treatment with unnecessary extraction of the upper and lower right first premolar teeth leading to asymmetric dental arches. The patient complained of dental crowding, an unaesthetic smile arch, and the maxillary and mandibular dental midline deviation. The proposed treatment plan goals were decrowding and correction of both arches asymmetry and achievement of coincident upper and lower dental midlines. The corrective treatment plan consisted of total arch mesialzation in both dental arches using an innovative TAD assisted jig. The overall treatment was accomplished in 28 months with significant improvement in facial aesthetic and reasonable periodontal status. This innovative clinical biomechanical setup of miniscrew-anchored sliding jig helped us achieve all the treatment goals (total dental arches mesialization, dental midline deviation correction, and ideal final aesthetic and occlusion) in a reasonable period of time. With proper planning, innovative designs based on TADs are effective alternatives in challenging cases such as uni or bilateral dental arches mesialization, and dental asymmetry correction.
\end{abstract}

Keywords: miniscrews; orthodontics; midline; asymmetry.

(C) 2020 by the authors. This article is an open-access article distributed under the terms and conditions of the Creative Commons Attribution (CC BY) license (https://creativecommons.org/licenses/by/4.0/).

\section{Introduction}

Orthodontic malpractice is a common entity in the field of dentistry due to an improper treatment plan. Thus, the management of cases with previous improper orthodontic treatment has been within an 'orthodontist's daily armamentarium. This is more challenging when the previous treatment plan consisted of unilateral extractions ended up with asymmetric arches and dental midline deviation. Asymmetries are among the most burdening problems in orthodontics, especially when both dental arches are at fault. Correction of asymmetric arches appeals to elaborate biomechanical considerations and versatile devices to achieve desired treatment goals without causing any iatrogenic or unwanted consequences. Previously, interarch elastics, asymmetrical extraction, space reopening for implant restoration, and unilateral distalization were the only treatment options for dental midline deviation correction. Nowadays, the versatility of TADs has considerably increased the spectrum of treatment choices planned by orthodontists. The use of TADs, absolute anchorage, has been achieved 
with optimal orthodontic mechanics, minimal unwanted reciprocal movement of the other teeth, and less dependence on the patient cooperation [1, 2].

In a review on various contemporary TAD-based devices, there is a considerable number of reports on the successful use of TADs with the purpose of distalizing the posterior teeth both unilateral and bilaterally. Whereas, few studies have reported the use of TADs for teeth mesialization with the purpose of dental arch asymmetry correction [3, 4]. In this study, we describe simultaneous unilateral mesialization of both asymmetric maxillary and mandibular dental arches to correct upper and lower midlines deviation using an innovative TAD-anchored sliding jig.

\section{Materials and Methods}

\subsection{Diagnosis.}

A 25-year-old male sought orthodontic treatment with a chief complaint of "crooked" dental midlines led to unpleasant esthetics. Deviation of the upper and lower midlines to the right was due to the extraction of the upper and lower right premolars in previous improper orthodontic treatment causing upper and lower dental arches yaw rotation to the right side (Figure 1).

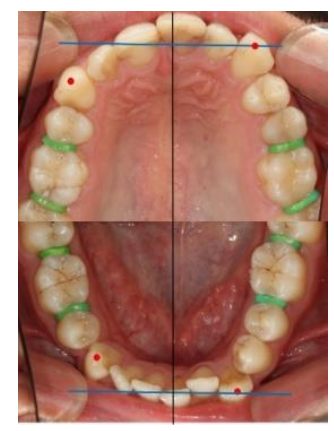

Figure 1. Occlusal view of the upper and lower dental arches yaw rotation.

The sagittal relationship between the upper and lower arches evinced a Class II unilateral molar and canine relationship, with the right side presenting complete Class I, while the left side presented molar half a cusp and canine full cusp Class II relationship. As regards soft-tissue facial profile, the patient had competent lips with a retruded position related to Eline. The patient's revealed approximately normal overjet and a slight tendency to deep bite (Figure 2).

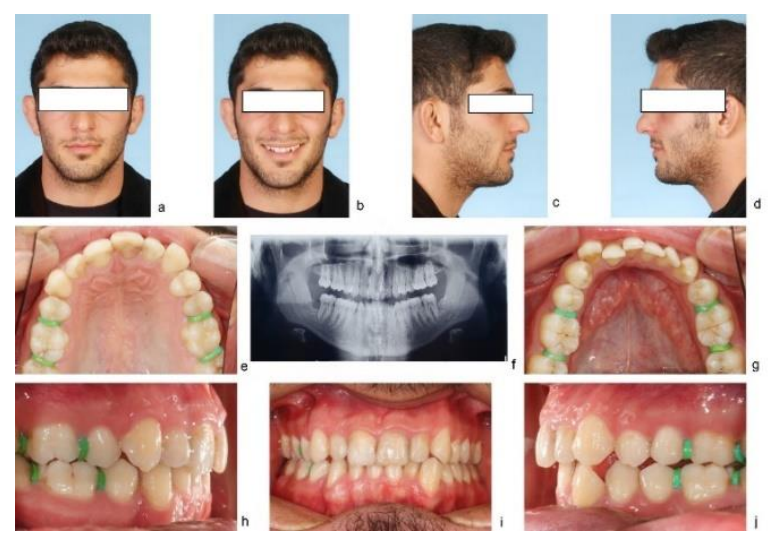

Figure 2. Extraoral views: frontal at rest (a), frontal at smile (b), right and left profile (c,d) intraoral views: upper occlusal (e), lower occlusal (g), right (h), left (j), frontal (i) and panoramic radiograph (f). 
The initial lateral cephalometric analysis showed skeletal cl I and normal inclination of the upper and lower incisors (Figure 3 and Table 1).

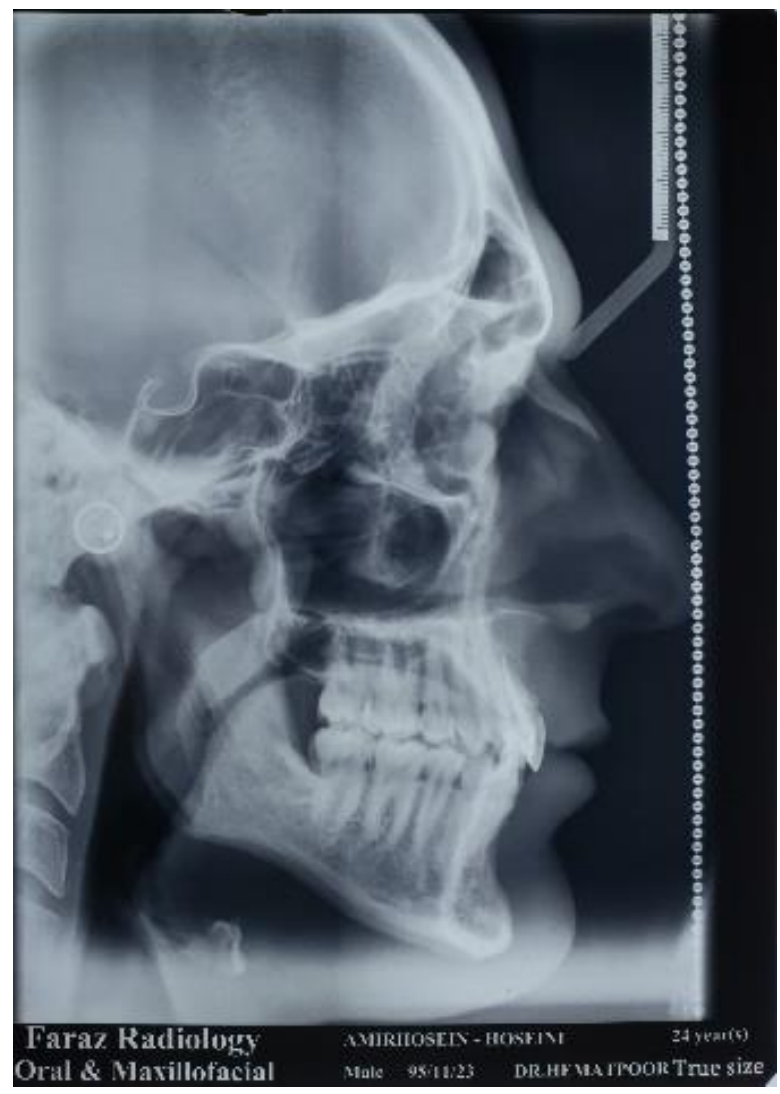

Figure 3. Initial lateral cephalogram.

Table 1. Pre-treatment values of cephalometric analysis.

\begin{tabular}{l|l}
$\begin{array}{l}\text { Cephalopetric } \\
\text { Variable }\end{array}$ & $\begin{array}{l}\text { Pre Treatment } \\
\text { (degrees) }\end{array}$ \\
\hline U1-SN & 100 \\
\hline L1-MeGo & 88 \\
\hline U1 to N-Pog & +2 \\
\hline L1 to N-Pog & +1 \\
\hline Interincisal A. & 137 \\
\hline U1-Palatal P. & 110 \\
\hline S-Go: N-Mex100 & 65 \\
\hline Pal-Go-Me(Basal A.) & 27 \\
\hline SN-MeGo & 34 \\
\hline SNA & 81 \\
\hline SNB & 78 \\
\hline ANB & 3 \\
\hline SN-Pog & 81 \\
\hline Wits & -1 \\
\hline Upper lip to E_line & 6 \\
\hline Lower lip to E_line & 4 \\
\hline Nasolabial angle & 102
\end{tabular}

\subsection{Treatment objectives.}

Orthodontic treatment planning objectives were to correct Class II unilateral malocclusion and also to correct upper and lower midline deviations. The sagittal dental relationship suggests that both dental arches need to be subjected to right to left mesialization to a coincide midline. The treatment goals included preserving the patient's profile, correction of the upper and lower dental arches asymmetry, coinciding the upper and lower arches 
midlines deviation to each other and to the facial midline, and obtaining bilateral canine neutroclusion and class I bilateral molar relationship.

\subsection{Treatment alternatives.}

The first treatment choice was space regaining for the right side dental implants, which were declined by the patient. The second choice was the extraction of the left upper and lowered first premolars, which was not adopted in this patient due to the patient's unwillingness to extractions and deep bite tendency. Also, premolar extraction was not adopted as an alternative in this patient because of his retruded lip position and profile. The third treatment choice was using skeletal anchorage in the radicular space of canine and second premolar area to protract the posterior teeth. Due to the amount of teeth mesalization, the susceptibility of the roots damage in the path of the teeth movements, and the necessity of several mini-screw replacements throughout the treatment, this option was also ruled out. At last, it was decided to apply two miniscrews in the palatal area and another on the lower right side of the arch distal to the last molar for total mesializtion of the arches without the need to mini_screw repositioning throughout the treatment period.

\subsection{Treatment progress.}

Prior to orthodontic treatment, all dental caries were removed and restored. The patient was also referred to an endodontist to confirm the health of the existing root canal treatment on the upper left central tooth. The third molars in all four quadrants were previously extracted. At the first orthodontic bonding appointment, only the maxillary arch was bonded. Pre-adjusted MBT metallic brackets (Master Series, American Orthodontics Ltd, Sheboygan, Wis, USA) with 0.022 slot size were utilized. 0.014"' superelastic nickel-titanium wire (austenitic active, preformed ovoid, superelastic archwire; 3M Unitek Corporation) was employed for initial alignment and leveling. At subsequent visits, the archwire was changed to 0.16 "' $\mathrm{NiTi}, .018$ "' NiTi and then $.019 \times .025$ "' rectangular (NiTi) until we could place $19 \times 25$ "' stainless steel as the working archwire.

Two mini-implants were placed in the anterior palatal area $(2 \mathrm{~mm} \times 11 \mathrm{~mm}$, in the area of the third palatal ruga, and $2 \mathrm{~mm} \times 9 \mathrm{~mm}$, about $8 \mathrm{~mm}$ distal to the first implant). Immediately after the placement of the mini implants, an impression was taken to adapt the hand-made sliding jig on the plaster working models.

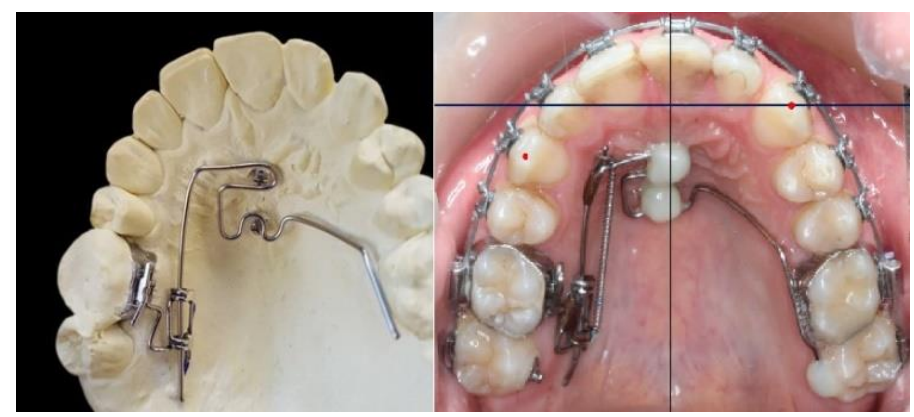

Figure 4. Designed jig for upper arch mesialization on the right side.

Incognito palatal sheaths were used for a stable connection to the molar bands. A sliding jig with an attached .043" wire was adapted to the palatal slope, and the jig was placed and activated with $500 \mathrm{~g}$ force level (nickel-titanium closed-coil spring) on the right side. The left 
side of the appliance was left passive. A heavy stabilizing wire framework in the lingual side of the teeth was adjusted to counteract with the lingo version side effect of the palatally mesialization force exerted on teeth and having a three-dimensional control on teeth movements. In order to minimize the device rocking, the jig was fixed to the second molar with a flowable composite (Figure 4).

After four months, brackets were placed on the mandibular arch to start the leveling and alignment using 0.014-inch NiTi archwire and archwire sequentially changed at the following appointments to accommodate the $19 \times 25$ "' stainless steel working archwire. A miniscrew $(1.6 \times 8$ mm, JB, Dual Top Anchor System; Jeil Medical, Seoul, Korea) was inserted on the right side, in the distal area of the buccal shelf. A special custom-made jig was designed using a $19 \times$ " 25 " stainless steel wire. In the posterior area, the jig was connected to the miniscrew and the archwire in the area between the second premolar and first molar, intimate to the second premolar bracket in order to allow a freeway for molar mesialization. Then, a loop was inserted in that region and bent upward and hooked on the archwire in the area between the second premolar and first molar to prevent upward and downward movement of the sliding jig. By using a crimpable hook-shaped lever arm crimped on the wire distal to the lower right second molar, total arch mesialization commenced using a $10 \mathrm{~mm}$ Sentalloy closed coil spring (Dentsply GAC Int., Bohemia, NY, USA) with heavy forced from the lever arch to the jig hook (Figure 5).

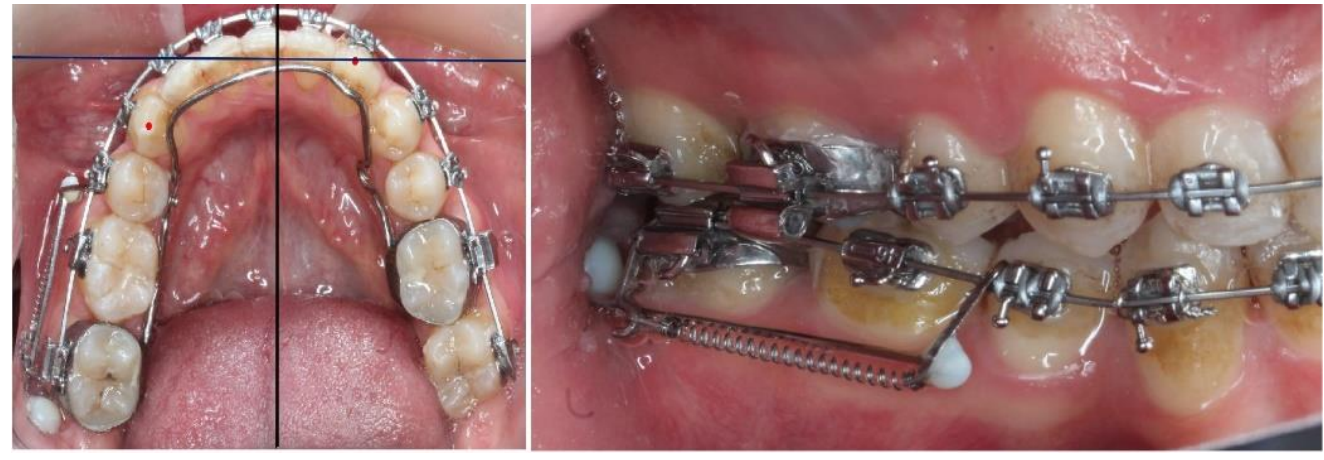

Figure 5. Special type of jig designed for posterior lower right mesialization.

During the treatment period, a lingual arch with adjustable U-loops was utilized in the lower arch to counteract with the buccoversion side effect of the mesialization force exerted from the buccal side on the teeth. Throughout the treatment time, sliding jigs and working wire and lingual arch U-loops adjustments were performed were needed to maintain the upper and lower arches coordination. Dental arches preparation was accomplished in 14 months. For retention, we used a bonded lingual retainer for six lower anterior teeth, and a Hawley retainer was used for the maxilla. The total duration of treatment was 28 months.

\section{Results and Discussion}

\subsection{Dental and soft tissue findings.}

Yaw correction was completely accomplished with sliding jigs in the upper and lower dental arches (Figures 6, 7, and 8).

The upper and lower dental midlines were coincident, and the smile arch revealed an acceptable aesthetic. The dental and facial midlines are coincident if not considering the patient's nose tip, which is deviated to the left. The upper and lower arch bases showed a 
skeletal class I relationship at the end of the treatment. Also, the final clinical evaluation demonstrated a normal class I canine and molar relationship. The soft tissue values related to lips with regard to E-line showed normal values at the end of the treatment.
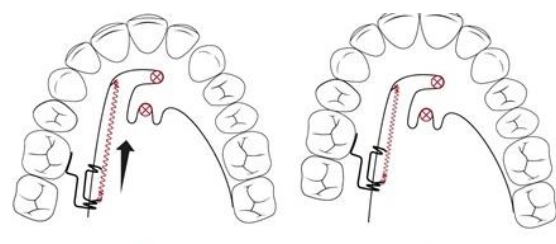

Figure 6. Schematic view of the upper dental arches mesialization.

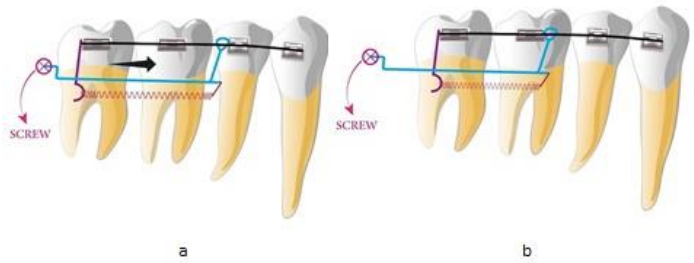

Figure 7. Schematic view of the lower dental arches mesialization.
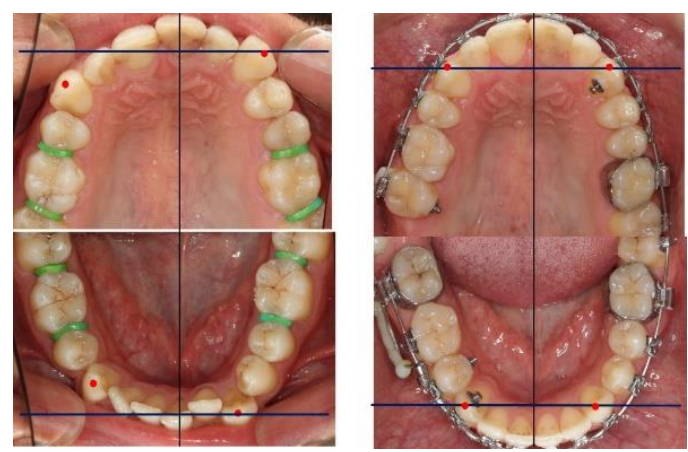

Figure 8. Yaw correction in the upper and lower arch.

\subsection{Cephalometric findings.}

Post-treatment panoramic radiograph showed root paralleling. Also, no significant evidence of root resorption or periodontal complication and loss of vitality in the mandibular and maxillary teeth was detected (Figure 9).

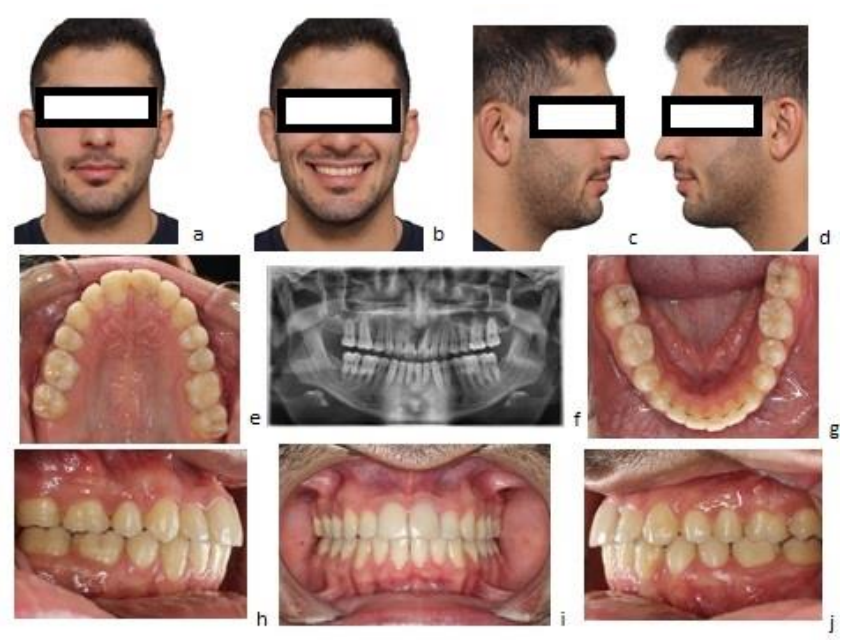

Figure 9. Extraoral views: frontal at rest (a), frontal at smile (b), right and left profile (c,d) intraoral views: upper occlusal (e), lower occlusal (g), right (h), left (j), frontal (i) and panoramic radiograph (f). 
The final lateral cephalometric analysis showed normal cephalometric variables values at the end of the treatment. Normal inclination of the upper and lower incisor teeth was obtained in the final cephalometric analysis (figure 10 and table 2).

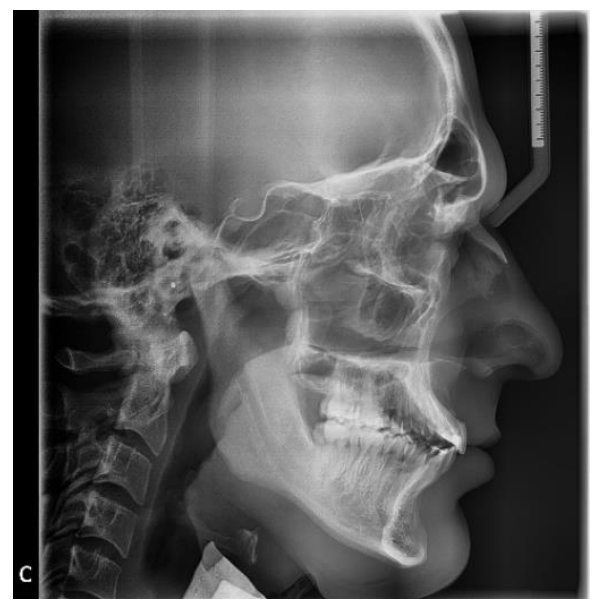

Figure 10. Final lateral cephalogram.

Upper incisors and lower incisors' relationship with regard to the N-Pog line increased at the end of the treatment. The skeletal chin position with regard to sella and cranial base demonstrated normal value at the end of the treatment. Nasolabial angle showed normal values in cephalometric analysis and in clinical evaluation at the end of the treatment. Overall, the patient's soft and hard tissue radiographic and cephalometric variables have improved at the end of the treatment.

Table 2. Post-treatment values of cephalometric analysis.

\begin{tabular}{l|l}
$\begin{array}{l}\text { Cephalopetric } \\
\text { variable }\end{array}$ & $\begin{array}{l}\text { Post Treatment } \\
\text { (degrees) }\end{array}$ \\
\hline U1-SN & 116 \\
\hline L1-MeGo & 97 \\
\hline U1 to N-Pog & +6 \\
\hline L1 to N-Pog & +4 \\
\hline Interincisal A. & 103 \\
\hline U1-Palatal P. & 126 \\
\hline S-Go: N-Mex100 & 65 \\
\hline Pal-Go-Me(Basal A.) & 27 \\
\hline SN-MeGo & 34 \\
\hline SNA & 82 \\
\hline SNB & 79 \\
\hline ANB & 3 \\
\hline SN-Pog & 81 \\
\hline Wits & 0 \\
\hline Upper lip to E_line & -5 \\
\hline Lower lip to E_line & -3 \\
\hline Nasolabial angle & 102
\end{tabular}

\subsection{Discussion.}

Midline correction in cases with previous unilateral tooth extraction history is one of the most challenging feats in orthodontics besides the other problems in oral such as oral cancer, bone defects, oral infections, oral skin, and so on [5-12]. This is more complicated when this midline deviation is caused by an iatrogenic improper previous orthodontic treatment. Unilateral extraction treatments may end up with compromising aesthetic results, therefore; suitable mechanics may be implemented to close space, with a caution against the excess collapse of the arch or deviation of the midline from ideal [12]. 
To manage an unsuccessful previous unilateral extraction case, the orthodontist must use his/her experience and common clinical sense to make the most suitable alternative between space reopening for implanting the previously extracted tooth or extraction of the other tooth/teeth to restore the occlusal balance and aesthetic goals. The treatment plan must meet the patient's expectations and manage the chief clinical complaint without imposing overtreatment or extending the treatment period. Multiple contributors play a rule in the treatment decision making, including technical complexity, therapeutic predictability, and clinician's experience and patient compliance, which determine the best therapeutic choice and, therefore, success. In this study, we presented a patient with a deviated midline due to previous asymmetric extractions retreated successfully using a novel TAD-based hand-made jig by unilateral total arch mesializaton in both upper and lower arches.

There are some studies in the literature reporting successful use of TADs for uni or bilateral molar distalization with the aim of midline correction [13-15] or class II correction [16]. Also, there are some reports of successful TAD-assisted unilateral posterior segment mesialization in a single arch (upper) with the aim of space closure in multiple missing cases [17] or bilateral posterior mesialization only in the upper arch using Mesialslider [18]. But, we found no clinical study in the literature pertaining to simultaneous en masse arch mesialization in both upper and lower arch and especially in the lower arch per se. Our study utilized a new technique, not reported previously in the literature, to successfully derotate two deviated dental arches into symmetric ones by a right to left total mesialization in both arches, which is the challenging and novel point of the case.

The result of some researches revealed that molar mesialization is challenging because following tooth mesialization, there are probable complications such as periodontal support loss, extrusion, and rotation of the mesialized segments and non-intended alterations of the occlusal force distribution [19-22].

In contrast, the result of a recent study showed that molar mesialization by TADs contributes to diminished treatment duration, guided tooth movement, and less adverse effects on the alveolar ridge and associated periodontium [23]. This result is in line with our study that revealed no adverse effect on periodontium in both radiographic and clinical final evaluations.

A recent in-vitro finite element study simulated teeth movement and biomechanically analyzed total mandibular arch mesialization. The authors reported that due to the sensitivity of this movement, accuracy in choosing the best force angulation and biomechanics is crucial to obtain ideal tooth movements with minimal side effects such as unintended occlusal plane alteration and tipping [24]. In this study, we reported successful mandibular and maxillary arches total mesialization with precisely designed TAD-based technique to exert a proper force to the center of rotation and meet both treatment goals and biomechanical considerations with no detrimental side effects.

Also, a three-dimensional study reported that skeletally anchored molar mesialization by Mesialslider could preserve the arch form and lead to a premolar width without considerably clinical anchorage loss [25]. This is in line with our study results that showed with a heavy hand-made framework in the upper palatal area (similar to the concept of Mesialslider appliance) and lingual arch in the lower arch, the management of guided molars mesialization pathway in three-dimensions was predictably possible by using TADs.

Another well-thought point of the treatment planning, in this case, is the insertion site of the miniscrews considering the anchorage value and location. The buccal shelf was adopted for the lower arch to invade any probable roots contact and the need for several miniscrew 
replacements. Although the buccal shelf is one of the commonly used sites for molar distalization, this area was not reported previously in the literature to be used for a mandibular arch, posterior mesialization.

The median anterior palatal region, with a safe distance from the incisive canal and incisor roots, was selected for the upper arch miniscrews insertion. This region is preferred by many clinicians for miniscrew insertion because it is more advantageous rather than the paramedian area in several aspects such as the reduced risk of incisors root damage, the possibility of miniscrew insertion in a more anterior region with more available bone, and also, coupling in the line of force for appropriate sagittal (mesial in this case) and vertical movements $[26,27]$.

In this study report, second molar mesialization was conducted by a single TAD inserted in the buccal side of the missing first premolar alveolar process, which required excessive considerations to compensate for the molar mesial tipping and crossbite effect caused by mesiobuccal exerted force line [28]. In the study, we designed a heavy metal framework in the palatal area and lingual arch in the lower arch with accurately and frequently adjusted heavy working wires in the buccal to precisely guide the mesialization movement pathway and prevent any unwanted teeth movements during molar mesialization.

\section{Conclusions}

Technology advanced has contributed significantly to the field of treatment planning in dentistry and Orthodontics. Application of TADs in modern dentistry has revolutionized treatment approaches to reach ideal treatment results. The history of past treatment with an incorrect diagnosis may entail some iatrogenic side effects which impose a more complicated and lengthy future treatment, which are sometimes impossible or burdening to compensate with routinely used conventional orthodontic appliances. Innovative application of TADs could simplify the modification of iatrogenically skewed dental arches with desirable aesthetics. Using TADs have provided a more predictable and less invasive treatment results compared to other compelling treatment plan alternatives.

\section{Funding}

This research received no external funding.

\section{Acknowledgments}

The authors would like to thank the Department of Orthodontics, School of dentistry, Shahid Beheshti, and Islamic Azad University of Medical Sciences, Tehran, Iran.

\section{Conflicts of Interest}

The authors declare no conflict of interest.

\section{References}

1. Kircelli, B.H.; Pektas, Z.O.; Kircelli, C. Maxillary molar distalization with a bone-anchored pendulum appliance. Angle Orthod 2006, 76, 650-9.

2. Arcuri, C.; Muzzi, F.; Santini, F.; Barlattani, A.; Giancotti, A. Five years of experience using palatal miniimplants for orthodontic anchorage. J. Oral Maxillofac. Surg 2007, 65, 2492-2497, https://doi.org/10.1016/j.joms.2007.06.651. 
3. Kılınç, D.D.; Sayar, G. Various Contemporary Intraoral Anchorage Mechanics Supported with Temporary Anchorage Devices. Turk J Orthod 2016, 29, 109-113.

4. Wilmes, B.; Nanda, R.; Nienkemper, M.; Ludwig, B.; Drescher, D. Correction of upper-arch asymmetries using the Mesial-Distalslider. J Clin Orthod 2013, 47, 648-655.

5. Tahmasebi, E.; Alikhani, M.; Yazdanian, A.; Yazdanian, M.; Tebyanian, H.; Seifalian, A. The current markers of cancer stem cell in oral cancers. Life Sci 2020, 249, https://doi.org/10.1016/j.lfs.2020.117483.

6. Soufdoost, R.S.; Mosaddad, S.A.; Salari, Y.; Yazdanian, M.; Tebyanian, H.; Tahmasebi, E.; Yazdanian, A.; Karami, A.; Barkhordari, A. Surgical Suture Assembled with Tadalafil/Polycaprolactone Drug-Delivery for Vascular Stimulation Around Wound: Validated in a Preclinical Model. Biointerface Res Appl Chem 2020, 10, 6317-6327, https://doi.org/10.33263/BRIAC105.63176327.

7. Zolfaghar, M.; Amoozegar, M.A.; Khajeh, K.; Babavalian, H.; Tebyanian, H. Isolation and screening of extracellular anticancer enzymes from halophilic and halotolerant bacteria from different saline environments in Iran. Mol. Biol. Rep 2019, 46, 3275-3286, https://doi.org/10.1007/s11033-019-04787-7.

8. Mosaddad, S.A.; Tahmasebi, E.; Yazdanian, A.; Rezvani, M.B.; Seifalian, A.; Yazdanian, M.; Tebyanian, H. Oral microbial biofilms: an update. Eur J Clin Microbiol 2019, 38, 2005-2019, https://doi.org/10.1007/s10096-019-03641-9.

9. Karami, A.; Tebyanian, H.; Barkhordari, A.; Motavallian, E.; Soufdoost, R.S.; Nourani, M.R. Healing effects of ointment drug on full-thickness wound. C. R. Acad. Bulg. Sci 2019, 72, 123-129.

10. Khomarlou, N.; Aberoomand-Azar, P.; Lashgari, A.P.; Tebyanian, H.; Hakakian, A.; Ranjbar, R.; Ayatollahi, S.A. Essential oil composition and in vitro antibacterial activity of Chenopodium album subsp. striatum. Acta Biologica Hungarica 2018, 69, 144-155, https://doi.org/10.1556/018.69.2018.2.4.

11. Shakeri, F.; Tebyanian, H.; Karami, A.; Babavalian, H.; Tahmasbi, M.H. Effect of Topical Phenytoin on Wound Healing. Trauma. Mon 2017, 22, https://doi.org/10.9790/0853-150902161164.

12. Dahiya, G.; Masoud, A.I.; Viana, G.; Obrez, A.; Kusnoto, B.; Evans, C.A. Effects of unilateral premolar extraction treatment on the dental arch forms of Class II subdivision malocclusions. Am J Orthod Dentofacial Orthop 2017, 152, 232-241, https://doi.org/10.1016/j.ajodo.2017.04.013.

13. Lim, J.K.; Jeon, H.J.; Kim, J.H. Molar distalization with a miniscrew-anchored sliding jig. J Clin Orthod 2011, 45, 368-77.

14. Shimazaki, K.; Kanno, Z.; Ono, T. Alternative approach using miniscrew-anchored sliding jig to correct maxillary midline deviation in a patient with unilateral missing premolar. APOS Trends Orthod 2019, 9, 111-116, https://doi.org/10.25259/APOS-57-2019.

15. Ma, Q.L.; Conley, R.S.; Wu, T.; Li, H. Asymmetric molar distalization with miniscrews to correct a severe unilateral Class III malocclusion. Am J Orthod Dentofacial Orthop 2016, 149, 729-39, https://doi.org/10.1016/j.ajodo.2015.07.042.

16. Papadopoulos, M.A. Effective Treatment of Class II Malocclusion with the TAD-supported amda ${ }^{\circledR}$. In: Temporary Anchorage Devices in Clinical Orthodontics. 2020; pp. 153-160, https://doi.org/10.1002/9781119513636.ch17.

17. Gedrange, T.; Boening, K.; Harzer, W. Orthodontic implants as anchorage appliances for unilateral mesialization: A case report. Quintessence Int 2006, 37.

18. Wilmes, B.; Nienkemper, M.; Nanda, R.; Lübberink, G.; Drescher, D. Palatally anchored maxillary molar mesialization using the mesialslider. J Clin Orthod 2013, 47, 172-179.

19. Jacobs, C.; Jacobs-Müller, C.; Luley, C.; Erbe, C.; Wehrbein, H. Orthodontic space closure after first molar extraction without skeletal anchorage. J Orofac Orthop 2011, 72, 51-60, https://doi.org/10.1007/s00056010-0007-y.

20. Papadopoulos, M.A., Skeletal Anchorage in Orthodontic Treatment of Class II Malocclusion E-Book: Contemporary applications of orthodontic implants, miniscrew implants and mini plates. Elsevier Health Sciences. 2014, https://doi.org/10.1016/C2009-0-63214-1.

21. Winkler, J.; Göllner, N.; Göllner, P.; Pazera, P.; Gkantidis, N. Apical root resorption due to mandibular first molar mesialization: a split-mouth study. Am J Orthod Dentofac 2017, 151, 708-717, https://doi.org/10.1016/j.ajodo.2016.12.005.

22. Kuroda, S.; Tanaka, E. Risks and complications of miniscrew anchorage in clinical orthodontics. Jpn Dent Sci Rev 2014, 50, 79-85, https://doi.org/10.1016/j.jdsr.2014.05.001.

23. Rivis, O.; Potapchuk, A.; Goncharuk-Khomyn, M.; Bokoch, A. Use of Mini-Implant Anchorage For Second Molar Mesialization: Comprehensive Approach For Treatment Efficiency Analysis. Pesquisa Brasileira em Odontopediatria e Clínica Integrada 2019, 20, https://doi.org/10.1590/pboci.2020.018.

24. Kawamura, J.; Park, J.H.; Kojima, Y.; Kook, Y.A.; Kyung, H.M.; Chae, J.M. Biomechanical analysis for total mesialization of the mandibular dentition: A finite element study. Orthod Craniofac Res 2019, 22, 329336, https://doi.org/10.1111/ocr.12331.

25. Becker, K.; Wilmes, B.; Grandjean, C.; Vasudavan, S.; Drescher, D. Skeletally anchored mesialization of molars using digitized casts and two surface-matching approaches. J Orofac Orthop 2018, 79, 11-18, https://doi.org/10.1007/s00056-017-0108-y.

26. Wilmes, B.; Ludwig, B.; Vasudavan, S.; Nienkemper, M.; Drescher, D. The T-Zone: Median vs. Paramedian Insertion of Palatal Mini-Implants. J Clin Orthod 2016, 50, 543-551. 
27. Tahmasebi, E.; Alam, M.; Yazdanian, M.; Tebyanian, H.; Yazdanian, A.; Seifalian, A.; Mosaddad, S.A. Current biocompatible materials in oral regeneration: a comprehensive overview of composite materials. J Mater Res Technol 2020, 9, 11731-11755, https://doi.org/10.1016/j.jmrt.2020.08.042.

28. Marusamy, K.O.; Ramasamy, S.; Wali, O. Molar protraction using miniscrews (temporary anchorage device) with simultaneous correction of lateral crossbite: An orthodontic case report. J Int Soc Prev Community Dent 2018, 8, 271-276, https://doi.org/10.4103/jispcd.JISPCD_447_17. 